\title{
ILL-POSEDNESS FOR THE NONLINEAR SCHRÖDINGER EQUATION WITH QUADRATIC NON-LINEARITY IN LOW DIMENSIONS
}

\author{
TSUKASA IWABUCHI AND TAKAYOSHI OGAWA
}

\begin{abstract}
We consider the ill-posedness issue for the nonlinear Schrödinger equation with a quadratic nonlinearity. We refine the Bejenaru-Tao result by constructing an example in the following sense. There exist a sequence of time $T_{N} \rightarrow 0$ and solution $u_{N}(t)$ such that $u_{N}\left(T_{N}\right) \rightarrow \infty$ in the Besov space $B_{2, \sigma}^{-1}(\mathbb{R})(\sigma>2)$ for one space dimension. We also construct a similar ill-posed sequence of solutions in two space dimensions in the scaling critical Sobolev space $H^{-1}\left(\mathbb{R}^{2}\right)$. We systematically utilize the modulation space $M_{2,1}^{0}$ for one dimension and the scaled modulation space $\left(M_{2,1}^{0}\right)_{N}$ for two dimensions.
\end{abstract}

\section{INTRODUCTION}

We consider the ill-posedness issue of the initial value problem for the nonlinear Schrödinger equations with the quadratic nonlinearity:

$$
\left\{\begin{array}{lr}
i \partial_{t} u+\Delta u=u^{2}, & t \in \mathbb{R}, x \in \mathbb{R}^{n}, \\
u(0, x)=\phi(x), & x \in \mathbb{R}^{n} .
\end{array}\right.
$$

It is well known that the Cauchy problem (1.1) is time locally well-posed in the class $C\left([0, T) ; L^{2}\left(\mathbb{R}^{n}\right)\right)$ when $n=1,2,3$ by Tsutsumi [19] and $n=4$ by CazenaveWeissler [4] (see also Ginibre-Velo [8] and Kato [9]). For the quadratic nonlinearity, the well-posedness of the problem (1.1) is established in the Sobolev space $H^{s}\left(\mathbb{R}^{n}\right)$ with the negative regularity index $s \leq 0$. More precisely the well-posedness was proved in $H^{s}\left(\mathbb{R}^{n}\right)$ with $s>-3 / 4$ by Kenig-Ponce-Vega 10 for $n=1$, and Colliander-Delort-Kenig-Staffilani [5] for $n=2$. Bejenaru-Tao [2] improved the result when $n=1$ in the class $H^{s}$ with $s \geq-1$. For $n=2$, it was also improved by Bejenaru-de Silva [1] in $H^{s}$ with $s>-1$. Those results can be summarized as the following propositions:

Proposition $1.1([2,13])$. Let $s \geq-1$ and $n=1$. Then for any $\phi \in H^{s}\left(\mathbb{R}^{1}\right)$, there exists a local solution $u \in C\left([0, T) ; H^{s}\left(\mathbb{R}^{1}\right)\right)$ and the solution depends on the initial data continuously. Namely the initial value problem (1.1) is time locally well-posed. If $s<-1$, then the continuous dependence on the inital data generally fails.

The well-posedness result in two space dimensions is also known:

Proposition $1.2([1,13])$. Let $s>-1$ and $n=2$. Then for any $\phi \in H^{s}\left(\mathbb{R}^{2}\right)$, there exists a local solution $u \in C\left([0, T) ; H^{s}\left(\mathbb{R}^{2}\right)\right)$ and the solution depends on the initial data continuously. Namely the initial value problem (1.1) is locally well-posed.

Received by the editors May 24, 2012 and, in revised form, October 22, 2012.

2010 Mathematics Subject Classification. Primary 35Q55. 
When we consider a critical space for the time local well-posedness, the scaling invariance for the equation is an important factor for finding the threshold scale. For a solution $u(t, x)$ to the equation (1.1), the scaled function $u_{\lambda}(t, x)=\lambda u\left(\lambda^{2} t, \lambda x\right)$ also satisfies the same equation. Then the invariant homogeneous Sobolev space is $\dot{H}^{s}$ is given by $s=s_{*} \equiv \frac{n}{2}-2$ under the above scaling, and it is one of the possible thresholds for the time local well-posedness to the Cauchy problem (1.1). This is the simplest natural observation on the critical space for the time local well-posedness and ill-posedness on the initial value problem (1.1). However, it is not known whether this scaling argument remains valid or not. Indeed, for some other nonlinear partial differential equations, a different situation can be observed in some of the literature (see for instance Ponce-Sideris [17]).

Our purpose of this paper is twofold. One is to specify the critical Sobolev space between the well-posedness and the ill-posedness not only by the regularity index nor regularity scale in the Sobolev space but by a finer index involving the interpolation space. The other purpose is to show that the threshold space for the well-posedness and ill-posedness is different from the space that one may expect from the scaling argument if the space dimension is one. We then make it clear that the above well-posedness results for both $n=1$ and $n=2$ are indeed sharp and illustrate the explicit reason why the threshold space in one dimensional critical space does not coincide with the scaling critical space.

Before showing our main result, we recall some definitions for the Besov spaces.

Definition (The inhomogeneous Besov spaces). Let $\left\{\psi_{j}\right\}_{j}$ be the Littlewood-Paley dyadic decomposition of the unity; namely it satisfies $\widehat{\psi}(\xi) \in C_{0}^{\infty}, \widehat{\psi_{j}}(\xi)=\widehat{\psi}\left(\xi / 2^{j}\right)$ for all $j \in \mathbb{Z}$. $\quad \sum_{j} \widehat{\psi_{j}}(\xi) \equiv 1$ if $\xi \neq 0$. Let $\tilde{\psi}$ be a smooth function such that $\widehat{\tilde{\psi}}(\xi)=1$ on $B_{1}(0)$ and $\operatorname{supp} \widehat{\tilde{\psi}} \subset B_{2}(0)$. Then for any $s \in \mathbb{R}, 1 \leq p, \sigma \leq \infty$, the inhomogeneous Besov space $B_{p, \sigma}^{s}=B_{p, \sigma}^{s}\left(\mathbb{R}^{n}\right)$ is given by

$$
B_{p, \sigma}^{s}\left(\mathbb{R}^{n}\right)=\left\{f \in \mathcal{S}^{*} ;\|f\|_{B_{p, \sigma}^{s}} \equiv\left(\|\tilde{\psi} * f\|_{p}^{\sigma}+\sum_{j \geq 0} 2^{s j \sigma}\left\|\psi_{j} * f\right\|_{p}^{\sigma}\right)^{1 / \sigma}<\infty\right\} .
$$

For the one space dimension case, the ill-posedness is shown in any Besov space larger than the scaling critical Sobolev space $H^{-1}$. Namely we show the following result:

Theorem 1.3. Let $n=1$. For any fixed $\sigma>2$, there exist a sequence of time $\left\{T_{N}\right\}_{N}$ with $T_{N} \rightarrow 0(N \rightarrow \infty)$ and a sequence of initial data $\left\{\phi_{N}\right\}_{N} \subset L^{2}(\mathbb{R})$ $(N=1,2, \cdots)$ such that the corresponding sequence of the solution $\left\{u_{N}\right\}_{N} \subset$ $C\left([0, T) ; L^{2}(\mathbb{R})\right)$ to (1.1) with $u_{N}(0)=\phi_{N}$ satisfies

$$
\lim _{N \rightarrow \infty}\left\|\phi_{N}\right\|_{B_{2, \sigma}^{-1}} \rightarrow 0, \quad \lim _{N \rightarrow \infty}\left\|u_{N}\left(T_{N}\right)\right\|_{B_{2, \sigma}^{-1}}=\infty .
$$

Remark. The relation between the Sobolev space and the Besov space is the following:

$$
H^{-1}(\mathbb{R}) \subset B_{2, \sigma}^{-1}(\mathbb{R}) \subset H^{s}(\mathbb{R}) \quad \text { for all } 2<\sigma \leq \infty \text { and } s<-1 .
$$

Therefore Theorem 1.3 is a refinement of the result by Bejenaru-Tao [2]. Besides, our result states that the solution immediately blows up at $t \simeq 0$ as $N \rightarrow \infty$ if the function space is larger than the threshold case. This shows that the ill-posedness 
of the equation is much stronger than the statement shown in [2]. Note that the solution in [2] simply shows discontinuity as the frequency parameter $N \rightarrow \infty$.

For the two dimensional case, we also show a similar result as in Theorem 1.3 as follows.

Theorem 1.4. Let $n=2$. For any fixed $s \leq-1$, there exist a sequence of time $\left\{T_{N}\right\}_{N}$ with $T_{N} \rightarrow 0(N \rightarrow \infty)$ and the initial data $\left\{\phi_{N}\right\}_{N} \subset L^{2}\left(\mathbb{R}^{2}\right)(N=1,2, \cdots)$ such that the corresponding sequence of the solution $\left\{u_{N}\right\}_{N} \subset C\left([0, T) ; L^{2}\left(\mathbb{R}^{2}\right)\right)$ to (1.1) with $u_{N}(0)=\phi_{N}$ satisfies

$$
\lim _{N \rightarrow \infty}\left\|\phi_{N}\right\|_{H^{s}} \rightarrow 0, \quad \lim _{N \rightarrow \infty}\left\|u_{N}\left(T_{N}\right)\right\|_{H^{s}}=\infty .
$$

Remark. We should note that in the above two theorems, the solution $u_{N}$ is considered in $C\left(\left[0, \tilde{T}_{N}\right) ; L^{2}\left(\mathbb{R}^{n}\right)\right)$, where $\tilde{T}_{N}$ denotes the life span of the solution $u_{N}$, and $T_{N}<\tilde{T}_{N}$ for all $N$. Note that the equation is time locally well-posed in the class $L^{2}\left(\mathbb{R}^{n}\right)$. Therefore in those results, the sequence is chosen to be satisfied as $\left\|u_{N}\left(T_{N}\right)\right\|_{L^{2}} \rightarrow \infty$ as $N \rightarrow \infty$. It is possible to show the same result for the nonlinear term $\bar{u}^{2}$ instead of $u^{2}$ in (1.1). On the other hand, other nonlinearity such as $|u|^{2},|u| \bar{u}$ or $|u| u$, the threshold space might be different from the above results. Indeed, $L^{2}\left(\mathbb{R}^{n}\right)=H^{0}\left(\mathbb{R}^{n}\right)$ would be the critical space for the gauge invariant nonlinearity $|u| u$ independent of the dimension (see Kenig-Ponce-Vega [11]).

For the nonlinear heat equations

$$
\left\{\begin{array}{lr}
\partial_{t} u-\Delta u=u^{2}, & t \in \mathbb{R}, x \in \mathbb{R}^{n}, \\
u(0, x)=\phi(x), & x \in \mathbb{R}^{n},
\end{array}\right.
$$

it is also possible to obtain the same results as our theorems. Indeed, by replacing the Schrödinger evolution group $e^{-i t|\xi|^{2}}$ with the heat semigroup $e^{-t|\xi|^{2}}$, our proof in Section 4 and Section 5 can be applicable to the proof for the above nonlinear heat equations, and it is possible to show the ill-posedness of the solution (1.2) in $B_{2, \sigma}^{-1}(\mathbb{R})(2<\sigma \leq \infty)$ and $H^{s}\left(\mathbb{R}^{2}\right)(s \leq-1)$. We should notice that the threshold for the well-posedness for (1.2) is also different from the scaling invariant space for heat equations in one space dimension. For other nonlinearity such as $\partial_{x} u^{2}$, the difference does not appear (see [3,6]).

To see a rough idea of the proof, we introduce a formal expansion of the solution to (1.1) by some small parameter $\varepsilon>0$ as

$$
u=U_{0}+\varepsilon U_{1}+\varepsilon^{2} U_{2}+\varepsilon^{3} U_{3}+\cdots .
$$

Then we regard the parameter $\varepsilon$ depending only on the inverse of the frequency parameter $N$ for the initial data. Namely we expand $\phi$ by

$$
\phi=\phi_{0}+\varepsilon \phi_{1}+\varepsilon^{2} \phi_{2}+\cdots
$$

by $\varepsilon \simeq N^{-1}$ and consider the term of the solution to (1.1) $U_{k}[\phi]$ as the term which may derive each term of the initial data $\phi_{k}$. If we introduce the initial data whose low frequency part is vanishing and has only some frequency component $\phi_{1}$, then $\phi=0+\varepsilon \phi_{1}+\varepsilon^{2} \times 0+\cdots$, and we find that $U_{0} \equiv 0$. Then, if $u$ satisfies the equation 
(1.1), we have by the terms of order $\varepsilon^{k}$ with $k=1,2,3$ that

$$
\begin{aligned}
& \varepsilon:\left\{\begin{array}{c}
\left(i \partial_{t}+\Delta\right) U_{1}=0, \\
U_{1}(0)=\phi,
\end{array}\right. \\
& \varepsilon^{2}:\left\{\begin{array}{c}
\left(i \partial_{t}+\Delta\right) U_{2}=U_{1} U_{1}, \\
U_{2}(0)=0,
\end{array}\right. \\
& \varepsilon^{3}:\left\{\begin{array}{c}
\left(i \partial_{t}+\Delta\right) U_{3}=\left(U_{1} U_{2}+U_{2} U_{1}\right), \\
U_{3}(0)=0,
\end{array}\right.
\end{aligned}
$$

respectively. In general, we have on the terms of order $k \geq 2$ that

$$
\left\{\begin{array}{c}
\left(i \partial_{t}+\Delta\right) U_{k}=\sum_{k_{1}+k_{2}=k, k_{1}, k_{2} \geq 1} U_{k_{1}} U_{k_{2}}, \\
U_{k}(0)=0
\end{array}\right.
$$

formally. Therefore it is natural to introduce $\left\{U_{k}[\phi]\right\}_{k=1}^{\infty}$ by recurrence induction

$$
\left\{\begin{array}{l}
U_{1}[\phi](t):=e^{i t \Delta} \phi, \\
U_{k}[\phi](t):=\sum_{k_{1}+k_{2}=k, k_{1}, k_{2} \geq 0} \int_{0}^{t} e^{i(t-\tau) \Delta} U_{k_{1}}[\phi](\tau) U_{k_{2}}[\phi](\tau) d \tau
\end{array}\right.
$$

for any $k=1,2,3, \cdots$.

In view of the expansion (1.3), we consider that

$$
u(t)=U_{0}[0](t)+\sum_{k=1}^{\infty} \varepsilon^{k} U_{k}[\phi](t)=\sum_{k=1}^{\infty} \varepsilon^{k} U_{k}[\phi](t)
$$

is a solution to (1.1) for initial data $\varepsilon \phi$.

Under such a formal expansion, we investigate that each term from the solution can converge or diverge as $t \rightarrow 0$ by some initial test functions. One simple test function is the monochromatic data that the Fourier transform is supported locally at particular frequency. We let such a test function as $\phi_{N}$ with $\operatorname{supp} \widehat{\phi_{N}}(\xi) \simeq$ $\left\{\xi ;|\xi| \simeq 2^{N}\right\}$ for $N=1,2 \cdots$. By those test functions, one may find that all the higher terms from the expansion $U_{k}\left[\phi_{N}\right], k \geq k_{0}$, remain bounded under $N \rightarrow \infty$, while some lower order terms such as $U_{2}\left[\varphi_{j}\right]$ blow up in the larger space $H^{s}$ with $s<s_{0}$ at $N \rightarrow \infty$ with $t \rightarrow 0$. Then fixing the expansion parameter $\varepsilon$ as a constant, we see the equation is ill-posed in the space $H^{s}$ for all $s \leq s_{0}$ or $s<s_{0}$, where $s_{0}$ is the threshold index for the well-posedness and the ill-posedness. For the higher space dimensions $n=2,3$, the threshold exponent $s_{0}$ coincides with the scaling critical case $s_{*}=\frac{n}{2}-2$. On the other hand, in the one dimensional case, there appears a low frequency saturation, and one cannot attain the well-posedness up to the scaling critical space, for instance, letting $\phi:=2^{\left(-s-\frac{n}{2}\right) N} \psi_{N}$, where $\psi_{N}$ is one component of the Littlewood-Paley decomposition and $\|\phi\|_{H^{s}}$ is independent of $N$. By the Duhamel formula, we write

$$
u(t)=e^{i t \Delta} \phi+\int_{0}^{t} e^{i(t-\tau) \Delta} u^{2} d \tau
$$


and approximate the solution by the linear part $u \simeq e^{i t \Delta} \phi_{N}$ to see that

$$
\begin{aligned}
\left\|\int_{0}^{t} e^{i(t-\tau) \Delta}\left(e^{i \tau \Delta} \phi\right)^{2} d \tau\right\|_{H^{s}} & =\left\|(1+|\xi|)^{s} \int_{\mathbb{R}^{n}} \widehat{\phi}(\xi-\eta) \widehat{\phi}(\eta) \frac{e^{2 i t(\xi-\eta) \cdot \eta}-1}{2 i(\xi-\eta) \cdot \eta} d \eta\right\|_{L_{\xi}^{2}} \\
& \simeq\left\|(1+|\xi|)^{s}\right\|_{L^{2}\left(|\xi| \leq 2^{N}\right)} 2^{\left(-s-\frac{n}{2}\right) N \cdot 2} 2^{-2 N} 2^{N n} \\
& \simeq \max \left\{1,2^{\left(s+\frac{n}{2}\right) N}\right\} \cdot 2^{(-2 s-2) N} \\
& \simeq \max \left\{2^{-2(s+1) N}, 2^{\left(-\left(s-\frac{n}{2}+2\right) N\right.}\right\} .
\end{aligned}
$$

The last term diverges as $N \rightarrow \infty$ if $s<-1$ for $n=1$ and $s<\frac{n}{2}-2$ for $n \geq 2$. This irrationality occurs from the scaling difference between the lower and higher frequency weight $\left(1+|\xi|^{2}\right)^{s / 2}$ in the definition of inhomogeneous Sobolev spaces $H^{s}$. On the other hand, one may think that this difficulty might be avoided if we apply the homogeneous Sobolev space $\dot{H}^{s}$. However the well-posedness in the homogeneous space would not be suitable for our problem because the exponent $s$ is negative and the low frequency restriction in such a space is much more stringent than the inhomogeneous space when the space dimension is one (cf. $s_{*}=\frac{n}{2}-2=$ $\left.-\frac{3}{2}\right)$.

For the other equations, we refer to the results by Molinet 14 and MolinetVento [15] on the sharp ill-posedness for $\mathrm{Kdv}$ and $\mathrm{mKdV}$ equations and $\mathrm{KdV}$ Burgers equations. We should also note that for the other nonlinearity $\langle\nabla\rangle^{\beta} u^{2}$, Oh-Stefanov [16] showed the local well-posedness in $H^{s}\left(\mathbb{R}^{1}\right)$ with $\beta \in\left[0, \frac{1}{2}\right)$ and $-1+\beta<s<\frac{1}{2}$. In view of the above observation, the optimal regularity for the well-posedness is not clear for this type of nonlinearity.

In what follows, we firstly justify the formal expansion (1.4) in the modulation space $M_{2,1}^{0}\left(\mathbb{R}^{n}\right)$, where the product of functions is well defined and hence the justification is rather straightforward. Then we show that there exists a solution in the modulation space that satisfies the statement of the above theorems in one and two space dimensions. In the course of the proof, we also use the scaled modulation space $\left(M_{2,1}\right)_{N}$, where the Fourier window function $\chi_{k}$ is scaled by the parameter $N$. Using this space $\left(M_{2,1}\right)_{N}$, the derivative loss can be avoided according to the good property of the modulation spaces, and we may construct the unstable example showing the equation is indeed ill-posed in the low space dimensions.

\section{Preliminaries}

Before we go into the proof, we introduce the interpolation spaces. For $s \in \mathbb{R}$, let $H^{s}=H^{s}\left(\mathbb{R}^{n}\right)$ be the inhomogeneous Sobolev space defined by

$$
\|f\|_{H^{s}}=\left\|\mathcal{F}\left[\langle\xi\rangle^{s} \widehat{f}\right]\right\|_{2}<\infty
$$

The homogeneous Sobolev space is analogously defined by $f \in \dot{H}^{s}$ if

$$
\|f\|_{H^{s}}=\left\|\mathcal{F}\left[|\xi|^{s} \widehat{f}\right]\right\|_{2}<\infty .
$$

Let $\left\{\psi_{j}\right\}_{j}$ be the Littlewood-Paley dyadic decomposition of the unity; namely it satisfies $\widehat{\psi}(\xi) \in C_{0}^{\infty}, \widehat{\psi_{j}}(\xi)=\widehat{\psi}\left(\xi / 2^{j}\right)$ for all $j \in \mathbb{Z}$. $\sum_{j} \widehat{\psi_{j}}(\xi) \equiv 1$ if $\xi \neq 0$.

Definition (The homogeneous Besov spaces). Let $\left\{\psi_{j}\right\}_{j}$ be the Littlewood-Paley dyadic decomposition of the unity. For any $s \in \mathbb{R}, 1 \leq p, \sigma \leq \infty$, the homogeneous 
Besov space $\dot{B}_{p, \sigma}^{s}=\dot{B}_{p, \sigma}^{s}\left(\mathbb{R}^{n}\right)$ is given by

$$
\dot{B}_{p, \sigma}^{s}\left(\mathbb{R}^{n}\right)=\left\{f \in \mathcal{S}^{*} ;\|f\|_{\dot{B}_{p, \sigma}^{s}} \equiv\left(\sum_{j \in \mathbb{Z}} 2^{s j \sigma}\left\|\psi_{j} * f\right\|_{p}^{\sigma}\right)^{1 / \sigma}<\infty\right\} .
$$

Definition (The modulation spaces). Let $\chi_{k}$ be the Fourier window function that satisfies

$$
\operatorname{supp} \widehat{\chi_{k}} \subset\left\{\xi \in \mathbb{R}^{n} \mid k_{j}-1 \leq \xi_{j} \leq k_{j}+1 \text { for } j=1,2, \cdots, n\right\}, \quad \sum_{k \in \mathbb{Z}^{n}} \widehat{\chi_{k}}(\xi) \equiv 1 .
$$

Then for any $s \in \mathbb{R}, 1 \leq p, \sigma \leq \infty$, the modulation space $M_{p, \sigma}^{s}=M_{p, \sigma}^{s}\left(\mathbb{R}^{n}\right)$ is given by

$$
M_{p, \sigma}^{s}\left(\mathbb{R}^{n}\right)=\left\{f \in \mathcal{S}^{*} ;\|f\|_{M_{p, \sigma}^{s}} \equiv\left(\sum_{k \in \mathbb{Z}^{n}}(1+|k|)^{s \sigma}\left\|\chi_{k} * f\right\|_{p}^{\sigma}\right)^{1 / \sigma}<\infty\right\} .
$$

We abbreviate $M_{p, \sigma}^{s}$ as $M_{p, \sigma}$ if $s=0$.

The following embedding is well known for the modulation spaces.

Proposition 2.1. $\quad$ (1) For $s \in \mathbb{R}, M_{2,2}^{s}=H^{s}$.

(2) $M_{2, \sigma} \subset M_{2, \rho}$ if $1 \leq \sigma \leq \rho \leq \infty$.

(3) $M_{p, 1}^{0} \subset L^{q}$ for all $1 \leq p \leq q \leq \infty$ (continuous embedding).

(4) For $f, g \in M_{2,1}, f g \in M_{2,1}$ and there exists a constant $C_{M}>0$ such that

$$
\|f g\|_{M_{2,1}} \leq C_{M}\|f\|_{M_{2,1}}\|g\|_{M_{2,1}} .
$$

Hence $M_{2,1}$ is the Banach algebra.

Proof of Proposition 2.1. The proof of the properties of the modulation space, one can find in the literature (see [7, 18, 20]). For the reader's convenience, we outline the proof.

(1) Since the Fourier window function $\chi_{k}$ for $k \in \mathbb{Z}^{n}$ makes the sequence $\left\{\chi_{k} * f\right\}_{k}$ almost orthogonal, it is easy to see that $\|f\|_{M_{2,2}} \simeq\|f\|_{2}$. Namely $M_{2,2}^{s}=H^{s}$.

(2) It is a direct consequence of the inclusion relation in the sequence spaces $\ell^{\sigma} \subset \ell^{\rho}$ for $\sigma \leq \rho$.

(3) By the triangle inequality,

$$
\|f\|_{p} \leq \sum_{k}\left\|\chi_{k} * f\right\|_{p}
$$

and $M_{p, 1} \subset L^{p}$. While for $1 / p+1 / p^{\prime}=1$,

$$
|f(x)| \leq \sum_{k}\left|\widetilde{\chi_{k}} * \chi_{k} * f\right| \leq \sum_{k}\left\|\widetilde{\chi_{k}}\right\|_{p^{\prime}}\left\|\chi_{k} * f\right\|_{p} \leq C\|f\|_{M_{p, 1}} .
$$

Two estimates (2.2) and (2.3) yield the result.

(4) The product formula can be obtained by the paraproduct formula

$$
\|f g\|_{M_{2,1}} \leq C\|f\|_{M_{\infty, 1}}\|g\|_{M_{2,1}}
$$

and the embedding $M_{2,1}\left(\mathbb{R}^{n}\right) \hookrightarrow M_{\infty, 1}\left(\mathbb{R}^{n}\right)$. 
For the scaling argument, the modulation space is not invariant. Therefore we need to introduce the rescaled modulation space. The size is dyadic, and let $\|\cdot\|_{\left(M_{2,1}\right)_{N}}$ be defined by

$$
\|u\|_{\left(M_{2,1}\right)_{N}}:=\sum_{k \in 2^{N} \mathbb{Z}^{n}}\|\widehat{u}\|_{L^{2}\left(Q\left(k, 2^{N}\right)\right)},
$$

where

$$
Q\left(k, 2^{N}\right):=\left\{\left(\xi_{1}, \cdots \xi_{n}\right) \in \mathbb{R}^{n} \mid \xi_{j} \in\left[k_{j}, k_{j}+2^{N}\right] \text { for } j=1, \cdots, n\right\}
$$

for $k=\left(k_{1}, k_{2}, \cdots, k_{n}\right) \in \mathbb{Z}^{n}$.

Corollary 2.2. For any $N>0$, let $f, g \in\left(M_{2,1}\right)_{N}$. Then

$$
\|u v\|_{\left(M_{2,1}\right)_{N}} \leq C_{M} 2^{\frac{n N}{2}}\|u\|_{\left(M_{2,1}\right)_{N}}\|v\|_{\left(M_{2,1}\right)_{N}} .
$$

\section{Asymptotic EXPANSION}

In this section, we show the asymptotic expansion (1.4) of the solution to (1.1) near $t=0$. In what follows, we show the expansion assuming that $\varepsilon$ is proportional to the time variable and we do not assume the smallness of the initial data such as $u(0)=\phi_{0}+\varepsilon \phi_{1}+\varepsilon^{2} \phi_{2}+\cdots$. To this end, we reformulate the expansion scheme starting from $k=0$ instead of $k=1$. In this case the first several terms of the expansion $U_{k}[\phi](t)$ should be given by the equations

$$
\begin{aligned}
& \varepsilon^{0}:\left\{\begin{array}{c}
\left(i \partial_{t}+\Delta\right) U_{0}=U_{0} U_{0}, \\
U_{0}=\phi_{0},
\end{array}\right. \\
& \varepsilon^{1}:\left\{\begin{array}{c}
\left(i \partial_{t}+\Delta\right) U_{1}=U_{0} U_{1}+U_{1} U_{0}, \\
U_{1}(0)=\phi_{1},
\end{array}\right.
\end{aligned}
$$

and

$$
U[\phi](t) \equiv \sum_{k=0}^{\infty} \varepsilon^{k} U_{k}[\phi](t)
$$

formally gives the solution for (1.1) with the initial data $u(0)=\phi_{0}+\varepsilon \phi_{1}+\varepsilon^{2} \phi_{2}+\cdots$. For our purpose, let $\phi_{k} \equiv 0$ for all $k \geq 0$ except $\phi_{1}$. Then the formal expansion can be reduced as follows:

$$
\begin{aligned}
& \varepsilon^{0}:\left\{\begin{array}{c}
\left(i \partial_{t}+\Delta\right) U_{0}=U_{0} U_{0}, \\
U_{0}=0,
\end{array}\right. \\
& \varepsilon^{1}:\left\{\begin{array}{c}
\left(i \partial_{t}+\Delta\right) U_{1}=U_{0} U_{1}+U_{1} U_{0}=0, \\
U_{1}(0)=\phi_{1},
\end{array}\right.
\end{aligned}
$$

and therefore we obtain

$$
U[\phi](t) \equiv \sum_{k=1}^{\infty} \varepsilon^{k} U_{k}[\phi](t)
$$


with $U_{1}[\phi](t)=e^{i t \Delta} \phi_{1}$. Once we obtain this version of the expansion, the formal expansion that appeared in the introduction can be justified in an analogous way. Namely, we show the following result.

Proposition 3.1. For $\phi \in M_{2,1}$, there exist a small $T>0$ and a unique local solution $u=u(t, x)$ in $C\left([0, T) ; M_{2,1}\right)$ of the initial value problem (1.1). Then the following expansion in $C\left((0, T) ; M_{2,1}\right)$ is satisfied: For any $0<\varepsilon \leq 1$,

$$
u(t)=\sum_{k=1}^{\infty} \varepsilon^{k} U_{k}[\phi](t),
$$

where

$$
\left\{\begin{array}{l}
U_{1}[\phi](t):=e^{i t \Delta} \phi \\
U_{k}[\phi](t):=\sum_{k_{1}+k_{2}=k, k_{1}, k_{2} \geq 1} \int_{0}^{t} e^{i(t-\tau) \Delta} U_{k_{1}}[\phi](\tau) U_{k_{2}}[\phi](\tau) d \tau
\end{array}\right.
$$

for $k=1,2,3, \cdots$.

To show Proposition 3.1 we recall the basic property for the free Schrödinger group.

Lemma 3.2. For $\phi \in M_{2,1}$, let $e^{i t \Delta} \phi$ be the free Schrödinger evolution group in $L^{2}$. Then

$$
\begin{gathered}
\left\|e^{i t \Delta} \phi\right\|_{M_{2,1}}=\|\phi\|_{M_{2,1}}, \\
\sup _{t \in[0, T)]}\left\|\int_{0}^{t} e^{i(t-\tau) \Delta} F(\tau) d \tau\right\|_{M_{2,1}} \leq\|F\|_{L^{1}\left(0, T ; M_{2,1}\right)} .
\end{gathered}
$$

The proof for (3.5) is immediate from the definition of the modulation space. The estimate for the inhomogeneous term (3.6) directly follows from (3.5) by the Minkowski inequality. For more detailed estimates, see, for instance, 20].

Proof of Proposition 3.1. We show the case $\varepsilon=1$. The other case is the corollary of this case. Let $I=[0, T)$, where $T$ is to be chosen properly small. Firstly we show that scheme (3.4) is well defined in a class $C\left([0, T) ; M_{2,1}\right)$. Let $M>0$ be $\|\phi\|_{M_{2,1}} \leq M$. Then by (3.5), we have $\left\|U_{0}[\phi]\right\|_{M_{2,1}} \leq M$. Given $k \geq 1$ we assume that for all $0 \leq \ell \leq k-1, \sup _{t \in I}\left\|U_{\ell}[\phi]\right\|_{M_{2,1}} \leq C_{M}^{\ell-1} M^{\ell} t^{\ell-1}$. Then by (3.6) and (2.1),

$$
\begin{aligned}
\sup _{t}\left\|U_{k}[\phi]\right\|_{M_{2,1}} & \leq C_{M} \sum_{k_{1}+k_{2}=k, k_{1}, k_{2} \geq 1} \int_{0}^{t}\left\|U_{k_{1}}[\phi](\tau)\right\|_{M_{2,1}}\left\|U_{k_{2}}[\phi](\tau)\right\|_{M_{2,1}} d \tau \\
& \leq C_{M} \sum_{k_{1}+k_{2}=k} \int_{0}^{t} C^{k_{1}} C_{M}^{k_{1}-1} M^{k_{1}} t^{k_{1}-1} \cdot C^{k_{2}} C_{M}^{k_{2}-1} M_{k_{2}} t^{k_{2}-1} d \tau \\
& \leq C_{M} \sum_{k_{1}+k_{2}=k} C_{M}^{k-2} M^{k} \frac{t^{k-1}}{k-1} \\
& \leq C_{M}^{k-1} M^{k} t^{k-1}
\end{aligned}
$$

where we abbreviate the summation $\sum_{k_{1}+k_{2}=k, k_{1}, k_{2} \geq 1}$ by $\sum_{k_{1}+k_{2}=k}$. Then we see that

$$
\sup _{t \in I}\left\|U_{k}[\phi]\right\|_{M_{2,1}} \leq C_{M}^{k-1} M^{k} t^{k-1} \text {. }
$$


Therefore we conclude that $U[\phi](t) \equiv \sum_{k=1}^{\infty} U_{k}[\phi](t)$ is well defined by

$$
\|U[\phi]\|_{L^{\infty}\left(I ; M_{2,1}\right)} \leq \sum_{k=1}^{\infty} \sup _{t \in I}\left\|U_{k}[\phi](t)\right\|_{M_{2,1}} \leq M \sum_{k=1}^{\infty} C_{M}^{k-1} M^{k-1} T^{k-1} \leq M
$$

under $T<C_{M}^{k-1} M^{-1}$. We note that

$$
\begin{aligned}
\sum_{k=1}^{\infty} U_{k}[\phi](t) & =U_{1}[\phi]+\sum_{k=2}^{\infty} \sum_{k_{1}+k_{2}=k} \int_{0}^{t} e^{i(t-\tau) \Delta} U_{k_{1}}[\phi](\tau) U_{k_{2}}[\phi](\tau) d \tau \\
& =U_{1}[\phi]+\sum_{k=2}^{\infty} \sum_{k_{1}=1}^{k-1} \int_{0}^{t} e^{i(t-\tau) \Delta}\left(U_{k_{1}}[\phi](\tau)\right)\left(U_{k-k_{1}}[\phi](\tau)\right) d \tau \\
& =U_{1}[\phi]+\sum_{k_{1}=1}^{\infty} \sum_{k=k_{1}+1}^{\infty} \int_{0}^{t} e^{i(t-\tau) \Delta}\left(U_{k_{1}}[\phi](\tau)\right)\left(U_{k-k_{1}}[\phi](\tau)\right) d \tau \\
& =U_{1}[\phi]+\int_{0}^{t} e^{i(t-\tau) \Delta}\left(\sum_{k_{1}=1}^{\infty} U_{k_{1}}[\phi](\tau)\right)\left(\sum_{k_{2}=1}^{\infty} U_{k_{2}}[\phi](\tau)\right) d \tau .
\end{aligned}
$$

This shows that $U[\phi](t)$ is subject to the problem (1.1) with the initial data $\phi$ for small time interval $[0, T)$.

\section{One dimensional Case}

In this section we show the ill-posedness Theorem 1.3 by using the asymptotic expansion (3.3).

Proof of Theorem 1.3. Let $\varphi \in \mathcal{S}(\mathbb{R})$ be an even function satisfying $\varphi \geq 0$ :

$$
\operatorname{supp} \varphi \subset\{\xi \in \mathbb{R}|| \xi \mid \leq 1\}, \quad \varphi(\xi)=1 \text { for }|\xi| \leq \frac{1}{2} .
$$

Then, we denote $\varphi_{j}^{+}, \varphi_{j}^{-}$by

$$
\varphi_{j}^{+}(\xi):=\varphi\left(\xi-2^{j}\right), \quad \varphi_{j}^{-}(\xi):=\varphi\left(\xi+2^{j}\right) .
$$

Then, for $0<\delta<1, N \in \mathbb{N}$, let $\phi_{N}$ be defined by

$$
\phi_{N}:=\frac{\log (N+1)}{N^{\frac{1}{2}}} \sum_{N \leq j \leq(1+\delta) N} 2^{j} \mathcal{F}^{-1}\left[\varphi_{j}^{+}+\varphi_{j}^{-}\right],
$$

and let $u_{N}$ be a solution to (1.1) for initial data $\phi_{N}$. Then, we see that there exists $C=C(\delta)>0$ such that for any $\sigma>2$,

$$
\left\|\phi_{N}\right\|_{\dot{B}_{2, \sigma}^{-1}} \leq C N^{-\frac{1}{2}+\frac{1}{\sigma}} \log (1+N) \rightarrow 0 \quad \text { as } N \rightarrow \infty .
$$

For the estimate of $U_{k}\left[\phi_{N}\right](k \geq 2)$, we show the following lemma.

Lemma 4.1. (1) For $|\xi| \leq \frac{1}{2}$ and $t \geq 2^{-2 N}$, it holds that

$$
\left|\widehat{U_{2}\left[\phi_{N}\right]}(t, \xi)\right| \geq c\{\log (1+N)\}^{2} .
$$


(2) There exist $C>0$ and $0<\delta<1$ such that

$$
\sup _{t \in(0, T)}\left\|U_{k}\left[\phi_{N}\right]\right\|_{M_{2,1}} \leq C^{k} C_{M}^{k-1} T^{k-1} 2^{k(1+\delta) N},
$$

for all $k=3,4, \cdots$, where $C_{M}>0$ is the constant that appeared in (2.1).

Proof of Lemma 4.1. To prove (4.1), we integrate $\tau$ variable firstly to get

$$
\begin{aligned}
& \left.\widehat{U_{2}\left[\phi_{N}\right.}\right](t, \xi) \\
& =\int_{0}^{t} e^{-(t-\tau)|\xi|^{2}} \int_{\mathbb{R}} e^{-i \tau|\xi-\eta|^{2}} \widehat{\phi_{N}}(\xi-\eta) e^{-i \tau|\eta|^{2}} \widehat{\phi_{N}}(\eta) d \eta d \tau \\
& =\int_{\mathbb{R}} \widehat{\phi_{N}}(\xi-\eta) \widehat{\phi_{N}}(\eta) \frac{1}{2 \eta \cdot(\eta-\xi)} d \eta-\int_{\mathbb{R}} \widehat{\phi_{N}}(\xi-\eta) \widehat{\phi_{N}}(\eta) \frac{e^{-2 i t \eta \cdot(\eta-\xi)}}{2 \eta \cdot(\eta-\xi)} d \eta \\
& \equiv I+I I .
\end{aligned}
$$

For the estimate of $I$ in the case $|\xi| \leq 1 / 2$,

$$
\begin{aligned}
|I| & =\frac{(\log (1+N))^{2}}{N} \\
& \times\left|\sum_{N \leq j \leq(1+\delta) N} 2^{2 j} \int_{\mathbb{R}}\left(\varphi_{j}^{+}(\xi-\eta) \varphi_{j}^{-}(\eta)+\varphi_{j}^{-}(\xi-\eta) \varphi_{j}^{+}(\eta)\right) \frac{1}{2 \eta \cdot(\eta-\xi)} d \eta\right| \\
& \geq \frac{c(\log (1+N))^{2}}{N} \sum_{N \leq j \leq(1+\delta) N} 2^{2 j} 2^{-2 j} \\
& \geq \delta c(\log (1+N))^{2} .
\end{aligned}
$$

For $I I$ in the case $|\xi| \leq 1 / 2$,

$$
e^{-2 i t \eta \cdot(\eta-\xi)}=\frac{\partial_{\eta} e^{-2 i t \eta \cdot(\eta-\xi)}}{-2 i t(2 \eta-\xi)},
$$

and by integration by parts in $\eta_{1}$, we get

$$
\begin{aligned}
|I I|= & \left|\int_{\mathbb{R}} \frac{e^{-2 i t \eta \cdot(\eta-\xi)}}{2 i t} \partial_{\eta_{1}}\left\{\frac{\widehat{\phi_{N}}(\xi-\eta) \widehat{\phi_{N}}(\eta)}{2(2 \eta-\xi) \eta(\eta-\xi)}\right\} d \eta\right| \\
\leq & \int_{\mathbb{R}} \frac{1}{2|t|} \cdot \frac{|2 \eta-\xi|^{2}+2|\eta \cdot(\eta-\xi)|}{2|2 \eta-\xi|^{2}|\eta|^{2}|\eta-\xi|^{2}}\left|\widehat{\phi_{N}}(\xi-\eta) \widehat{\phi_{N}}(\eta)\right| d \eta \\
& +\left|\int_{\mathbb{R}} \frac{e^{-2 i t \eta \cdot(\eta-\xi)}}{4 t\left(2 \eta_{1}-\xi_{1}\right) \eta \cdot(\eta-\xi)} \partial_{\eta_{1}}\left\{\widehat{\phi_{N}}(\xi-\eta) \widehat{\phi_{N}}(\eta)\right\} d \eta\right| \\
= & : I I_{1}+I I_{2} .
\end{aligned}
$$

Since we restrict ourselves to $|\xi| \leq 1 / 2$ and $t \geq 2^{-2 N}$, we have on $I I_{1}$,

$$
\begin{aligned}
I I_{1} \leq & \frac{C v(\log (1+N))^{2}}{N} \\
& \times \sum_{N \leq j \leq(1+\delta) N} \int_{\mathbb{R}} \frac{1}{2^{-2 N}} \frac{2^{2 j}}{2^{6 j}} 2^{2 j}\left(\varphi_{j}^{+}(\xi-\eta)+\varphi_{j}^{-}(\xi-\eta)\right)\left(\varphi_{j}^{+}(\eta)+\varphi_{j}^{-}(\eta)\right) d \eta \\
& \leq \frac{C(\log (1+N))^{2}}{N} \rightarrow 0
\end{aligned}
$$


as $N \rightarrow \infty$. On the estimate of $I I_{2}$, by the symmetry of $\varphi_{j}^{ \pm}$, we find

$\int_{\mathbb{R}} \frac{e^{-2 i t \eta \cdot(\eta-\xi)}}{4 t\left(2 \eta_{1}-\xi_{1}\right) \eta \cdot(\eta-\xi)} \partial_{\eta_{1}}\left\{\varphi_{j}^{+}(\xi-\eta) \varphi_{j}^{-}(\eta)+\varphi_{j}^{-}(\xi-\eta) \varphi_{j}^{+}(\eta)\right\} d \eta=0$ for any $j ;$ thus it follows that

$$
\begin{aligned}
I I_{2}=\mid \frac{(\log (1+N))^{2} 2^{j}}{N} & \sum_{N \leq j \leq(1+\delta) N} \int_{\mathbb{R}} \frac{e^{-2 i t \eta \cdot(\eta-\xi)}}{4 t\left(2 \eta_{1}-\xi_{1}\right) \eta \cdot(\eta-\xi)} \\
& \times \partial_{\eta_{1}}\left\{\varphi_{j}^{+}(\xi-\eta) \varphi_{j}^{-}(\eta)+\varphi_{j}^{-}(\xi-\eta) \varphi_{j}^{+}(\eta)\right\} d \eta \mid=0 .
\end{aligned}
$$

Therefore, we obtain (4.1).

We show (4.2) by induction. On the estimate in the case $k=1$, we have

$$
\sup _{t \in(0, T)}\left\|U_{1}\left[\phi_{N}\right]\right\|_{M_{2,1}} \leq \frac{C \log (N+1)}{N^{\frac{1}{2}}} \sum_{N \leq j \leq(1+\delta) N} 2^{j} \leq C 2^{(1+\delta) N} .
$$

In the case $k=2$, we have from the bilinear estimate in modulation spaces, Proposition 2.1 and (4.4):

$$
\begin{aligned}
\sup _{t \in(0, T)}\left\|U_{2}\left[\phi_{N}\right]\right\|_{M_{2,1}} & \leq \int_{0}^{T} \sup _{t \in(0, T)}\left\|\left(U_{1}\left[\phi_{N}\right]\right)^{2}\right\|_{M_{2,1}} d \tau \\
& \leq C_{M} T \sup _{t \in(0, T)}\left\|U_{1}\left[\phi_{N}\right]\right\|_{M_{2,1}}^{2} \\
& \leq C^{2} C_{M} T 2^{2(1+\delta) N} .
\end{aligned}
$$

Let $k \geq 3$ and assume

$$
\sup _{t \in(0, T)}\left\|U_{k}\left[\phi_{N}\right]\right\|_{M_{2,1}} \leq C^{\ell} C_{M}^{\ell-1} T^{\ell-1} 2^{\ell(1+\delta) N},
$$

for $\ell=1,2, \cdots, k-1$. Then, it follows from the bilinear estimate in modulation spaces and the assumption of the induction that

$$
\begin{aligned}
& \sup _{t \in(0, T)}\left\|U_{k}\left[\phi_{N}\right]\right\|_{M_{2,1}} \\
& \leq \sum_{k_{1}+k_{2}=k, k_{1}, k_{2} \geq 1} \sup _{t \in(0, T)} \int_{0}^{t}\left\|U_{k_{1}}\left[\phi_{N}\right](\tau) U_{k_{2}}\left[\phi_{N}\right](\tau)\right\|_{M_{2,1}} d \tau \\
& \leq C_{M} \sum_{k_{1}+k_{2}=k} \sup _{t \in(0, T)} \int_{0}^{t}\left\|U_{k_{1}}\left[\phi_{N}\right](\tau)\right\|_{M_{2,1}}\left\|U_{k_{2}}\left[\phi_{N}\right](\tau)\right\|_{M_{2,1}} d \tau \\
& \leq C_{M} \sum_{k_{1}+k_{2}=k} \sup _{t \in(0, T)} \int_{0}^{t} C^{k_{1}} C_{M}^{k_{1}-1} \tau^{k_{1}-1} 2^{k_{1}(1+\delta) N} C^{k_{2}} C_{M}^{k_{2}-1} \tau^{k_{2}-1} 2^{k_{2}(1+\delta) N} d \tau \\
& =C^{k} C_{M}^{k-1} T^{k-1} 2^{k(1+\delta) N} .
\end{aligned}
$$

Therefore, we obtain (4.2).

Proof of Theorem 1.3 concluded. By the expansion (3.3), we denote for each $N$ that

$$
u_{N}(t)=\sum_{k=1}^{\infty} U\left[\phi_{N}\right](t) \text {. }
$$


Then we have on the solution $u_{N}$ for initial data $\phi_{N}$ at time $t \leq 2^{-2(N-1)}$ :

$$
\begin{aligned}
\left\|u_{N}(t)\right\|_{B_{2, \sigma}^{-1}} & =\left(\left\|\widehat{u}_{N}(t)\right\|_{L^{2}\left(|\xi| \leq 2^{-1}\right)}^{\sigma}+\sum_{j \geq 0}\left\|\langle\xi\rangle^{-1} \widehat{u}_{N}(t)\right\|_{L^{2}\left(2^{j-1} \leq|\xi| \leq 2^{j+1}\right)}^{\sigma}\right)^{1 / \sigma} \\
& \geq\left\|\widehat{u}_{N}(t)\right\|_{L^{2}\left(|\xi| \leq 2^{-1}\right)} \\
& \geq\left\|\widehat{U_{2}[\phi](t)}\right\|_{L^{2}\left(|\xi| \leq 2^{-1}\right)}-\left\|\sum_{k \geq 3} \widehat{U_{k}[\phi](t)}\right\|_{L^{2}\left(|\xi| \leq 2^{-1}\right)} .
\end{aligned}
$$

We note in the last inequality that $\widehat{U_{1}[\phi]}(t, \xi)=0$ for $|\xi| \leq 2^{-1}$. On the estimate for $U_{2}$, we have from (4.1) that

$$
\left\|\widehat{U_{2}[\phi]}(t)\right\|_{L^{2}\left(|\xi| \leq 2^{-1}\right)} \geq c\{\log (N+1)\}^{2} \text { for } t \geq 2^{-2 N} .
$$

On the estimate of $\sum_{k \geq 3} U_{k}\left[\phi_{N}\right](t)$, we have from $M_{2,1}(\mathbb{R}) \hookrightarrow L^{2}(\mathbb{R})$ and (4.2) with $T_{N}=2^{-2 N}$ that

$$
\begin{aligned}
\sup _{t \leq T_{N}}\left\|\sum_{k \geq 3} \widehat{U_{k}[\phi]}(t)\right\|_{L^{2}\left(|\xi| \leq 2^{-1}\right)} & \leq C_{0} \sup _{t \leq T_{N}} \sum_{k \geq 3}\left\|\widehat{U_{k}[\phi]}(t)\right\|_{M_{2,1}} \\
& \leq C_{0} \sum_{k \geq 3} C^{k} C_{M}^{k-1} 2^{-2 N(k-1)} 2^{k(1+\delta) N} \\
& =C_{0} C 2^{(1+\delta) N} \sum_{k \geq 3}\left(C C_{M} 2^{-(1-\delta) N}\right)^{k-1} .
\end{aligned}
$$

Since $\delta<1$, for large $N$ with $C C_{M} 2^{-(1-\delta) N}<1$, we have

$$
\sup _{t \leq T_{N}}\left\|\sum_{k \geq 3} \widehat{U_{k}[\phi]}(t)\right\|_{L^{2}\left(|\xi| \leq 2^{-1}\right)} \leq C_{0} C^{-1} C_{M}^{-2} 2^{(-1+3 \delta) N} .
$$

Therefore by taking $\delta<\frac{1}{3}$ and $t \leq T_{N}=2^{-2 N}$ in (4.8), (4.9) and (4.10), we conclude that

$$
\lim _{N \rightarrow \infty}\left\|u_{N}\left(T_{N}\right)\right\|_{B_{2, q}^{-1}}=\infty
$$

\section{Two Dimensional CASE}

Proof of Theorem 1.4. We separate the proof into the cases $s<-1$ and $s=s_{*} \equiv$ -1 .

Ill-posedness in $H^{s}\left(\mathbb{R}^{2}\right)$ in the case $s<-1$. It is possible to show the illposedness in a similar way to the previous section. Indeed, let $\varphi \in \mathcal{S}\left(\mathbb{R}^{2}\right)$ be a radial function that satisfies $\varphi \geq 0$,

$$
\operatorname{supp} \varphi \subset\left\{\xi \in \mathbb{R}^{2}|| \xi \mid \leq 1\right\}, \quad \varphi(\xi)=1 \text { for }|\xi| \leq \frac{1}{2} .
$$

For the unit vector $e_{1}=(1,0)$ parallel to the $\xi_{1}$ axis, we denote $\varphi_{j}^{+}, \varphi_{j}^{-}$by

$$
\varphi_{j}^{+}(\xi):=\varphi\left(\xi-2^{j} e_{1}\right), \quad \varphi_{j}^{-}(\xi):=\varphi\left(\xi+2^{j} e_{1}\right) .
$$


Then, for $0<\delta<1, N \in \mathbb{N}$, let $\phi_{N}$ be defined by

$$
\phi_{N}:=\frac{\log (N+1)}{N^{\frac{1}{2}}} \sum_{N \leq j \leq(1+\delta) N} 2^{j} \mathcal{F}^{-1}\left[\varphi_{j}^{+}+\varphi_{j}^{-}\right],
$$

and let $u_{N}$ be a solution to (1.1) for initial data $\phi_{N}$. Then, we see that there exists $C=C(\delta)>0$ such that for $s<-1$,

$$
\left\|\phi_{N}\right\|_{H^{s}} \leq\left\|\phi_{N}\right\|_{\dot{B}_{2, \infty}^{-1}} \leq C N^{-\frac{1}{2}} \log (1+N) \rightarrow 0 \quad \text { as } N \rightarrow \infty .
$$

For the estimate of $U_{k}[f](k \geq 2)$, it is possible to show the same lemma in the case of $n=2$, and we can show the ill-posedness in the space $H^{s}\left(\mathbb{R}^{2}\right)(s<-1)$.

Ill-posedness in $H^{s_{*}}\left(\mathbb{R}^{2}\right)$ with $s_{*}=\frac{n}{2}-2=-1$. Let $\left\{\psi_{j}\right\}$ be the LittlewoodPaley dyadic decomposition and let initial data $\phi_{N, R}$ be defined by

$$
\phi_{N, R}:=R 2^{(-n+2) N} \psi_{N},
$$

where $N \in \mathbb{R}$ and $R>0$, and chosen by $R \rightarrow 0$ as $N \rightarrow \infty$. Then, we have for $s_{*}=-1$ that

$$
\begin{aligned}
\left\|\phi_{N, R}\right\|_{H^{s_{*}}} & =R\left\|\langle\xi\rangle^{\frac{n}{2}-2}\right\|_{L^{2}\left(|\xi| \simeq 2^{N}\right)} \leq C R 2^{-(n-2) N}\left(\int_{2^{N-1}}^{2^{N+1}} \frac{r^{n-1}}{\left(1+r^{2}\right)^{2-\frac{n}{2}}} d r\right)^{1 / 2} \\
& =C R 2^{-(n-2) N} 2^{(n-2) N}=C R \rightarrow 0 \quad \text { as } N \rightarrow \infty .
\end{aligned}
$$

From (5.2), we directly have

$$
\left\|U_{1}\left[\phi_{N, R}\right]\right\|_{H^{s_{*}}} \leq C R \rightarrow 0
$$

as well.

For the estimate of $U_{k}\left[\phi_{N, R}\right](k \geq 2)$, we use the modulation spaces $M_{2,1}\left(\mathbb{R}^{n}\right)$ whose norm depends on $N$. More precisely, we consider the isometric decomposition of modulation spaces $M_{2,1}\left(\mathbb{R}^{n}\right)$ whose size is dyadic and let $\|\cdot\|_{\left(M_{2,1}\right)_{N}}$ be defined by

$$
\|u\|_{\left(M_{2,1}\right)_{N}}:=\sum_{k \in 2^{N} \mathbb{Z}^{n}}\|\widehat{u}\|_{L^{2}\left(Q\left(k, 2^{N}\right)\right)},
$$

where

$$
Q\left(k, 2^{N}\right):=\left\{\left(\xi_{1}, \cdots, \xi_{n}\right) \in \mathbb{R}^{n} \mid \xi_{j} \in\left[k_{j}, k_{j}+2^{N}\right] \text { for } j=1, \cdots, n\right\}
$$

for $k=\left(k_{1}, k_{2}, \cdots, k_{n}\right) \in \mathbb{Z}^{n}$.

Lemma 5.1. (1) Let $n=2$. Then

$$
\left\|U_{2}\left[\phi_{N, R}\right]\right\|_{H^{-1}} \geq c t R^{2} 2^{2 N} N^{\frac{1}{2}} \text { for all } t \leq 2^{-2 N-10} .
$$

(2) Let $n \geq 2$. Then, we have

$$
\left\|U_{k}\left[\phi_{N, R}\right]\right\|_{\left(M_{2,1}\right)_{N}} \leq C^{k} R^{k} t^{k-1} 2^{\left(2 k-\frac{n}{2}\right) N} \quad \text { for all } t \leq 2^{-2 N-10},
$$

where $k=3,4, \cdots$. 
Proof of Lemma 5.1. To show (5.4), taking the Fourier transform and integrating on the time variable, we have

$$
\widehat{U_{2}\left[\phi_{N, R}\right]}(\xi)=\int_{\mathbb{R}^{2}} \widehat{\phi_{N, R}}(\xi-\eta) \widehat{\phi_{N, R}}(\eta) \frac{e^{2 i t(\xi-\eta) \cdot \eta}-1}{2 i(\xi-\eta) \cdot \eta} d \eta .
$$

There exists $c>0$ such that for any $t \leq 2^{-2 N-10}$ we have

$$
\operatorname{Re}\left[\frac{e^{2 i t(\xi-\eta) \cdot \eta}-1}{2(\xi-\eta) \cdot \eta}\right] \geq c t
$$

since

$$
2^{N-1} \leq|\xi-\eta|,|\eta| \leq 2^{N+1} \quad \text { and } \quad|2 i t(\xi-\eta) \cdot \eta| \leq 2^{-7} .
$$

Therefore, we have on the norm $\|\cdot\|_{H^{s_{*}}}$,

$$
\left\|U_{2}\left[\phi_{N, R}\right]\right\|_{H^{s_{*}}} \geq c t\left\|\langle\xi\rangle^{-1} \int_{\mathbb{R}^{2}} \widehat{\phi_{N, R}}(\xi-\eta) \widehat{\phi_{N, R}}(\eta) d \eta\right\|_{L^{2}} .
$$

Since the support of $\widehat{\phi_{N, R}}$ is included in $\left\{\xi \in \mathbb{R}^{2}|| \xi \mid \leq 2^{N+1}\right\}$ and the support of $\widehat{\phi_{N, R}} * \widehat{\phi_{N, R}}$ is included in $\left\{\xi|| \xi \mid \leq 2^{N+2}\right\}$, we have

$$
\begin{aligned}
\left\|U_{2}\left[\phi_{N, R}\right]\right\|_{H^{s_{*}}} & \geq c t\left\|\langle\xi\rangle^{s_{*}} \int_{\mathbb{R}^{2}} \widehat{\phi_{N, R}}(\xi-\eta) \widehat{\phi_{N, R}}(\eta) d \eta\right\|_{L^{2}\left(|\xi| \leq 2^{N+2}\right)} \\
& \geq c t\left\|\langle\xi\rangle^{-1} R^{2} 2^{2(-2+2) N} 2^{2 N}\right\|_{L^{2}\left(|\xi| \leq 2^{N+2}\right)} \\
& \geq c t R^{2} 2^{2 N}\left(\log 2^{N}\right)^{\frac{1}{2}} \\
& =c t R^{2} 2^{2 N} N^{\frac{1}{2}} .
\end{aligned}
$$

It is worth noting that we may also obtain the analogous estimate for the higher space dimension similarly to the proof of (5.4) as

$$
\begin{aligned}
\left\|U_{2}\left[\phi_{N, R}\right]\right\|_{H^{s_{*}}} & \geq c t\left\|\langle\xi\rangle^{s_{*}} \int_{\mathbb{R}^{2}} \widehat{\phi_{N, R}}(\xi-\eta) \widehat{\phi_{N, R}}(\eta) d \eta\right\|_{L^{2}\left(|\xi| \leq 2^{N+2}\right)} \\
& \geq c t\left\|\langle\xi\rangle^{s_{*}} R^{2} 2^{2\{-(n-2) N\}} 2^{n N}\right\|_{L^{2}\left(|\xi| \leq 2^{N+2}\right)} \\
& \geq c t 2^{s_{*} N} R^{2} 2^{-2(n-2) N} 2^{n N} 2^{\frac{n N}{2}} \\
& =c t R^{2} 2^{2 N} 2^{\left(s_{*}-\frac{n}{2}+2\right) N}=c t R^{2} 2^{2 N} .
\end{aligned}
$$

Here we notice that the difference between $n=2$ and the higher dimensional case is the extra factor $N^{1 / 2}$, which appears only for the case $n=2$. This factor plays a crucial role for proving the ill-posedness in the threshold space. 
We next show (5.5) for the $n$-dimensional case. For the estimate of $U_{1}\left[\phi_{N, R}\right]$ and $U_{2}\left[\phi_{N, R}\right]$, we have by $\left(M_{2,1}\right)_{N} \subset L^{2}$ and (2.4) that

$$
\begin{aligned}
\left\|U_{1}\left[\phi_{N, R}\right]\right\|_{\left(M_{2,1}\right)_{N}} \leq & C_{0} R 2^{(-n+2) N} 2^{\frac{n N}{2}}=C_{0} R 2^{\left(-\frac{n}{2}+2\right) N} \\
\left\|U_{2}\left[\phi_{N, R}\right]\right\|_{\left(M_{2,1}\right)_{N}} & \leq \int_{0}^{t}\left\|U_{1}\left[\phi_{N, R}\right]^{2}\right\|_{\left(M_{2,1}\right)_{N}} d \tau \\
& \leq C_{1} 2^{\frac{n N}{2}} \int_{0}^{t}\left\|U_{1}\left[\phi_{N, R}\right]\right\|_{\left(M_{2,1}\right)_{N}}^{2} d \tau \\
& \leq C_{1} 2^{\frac{n N}{2}} t C_{0}^{2} R^{2} 2^{2\left(-\frac{n}{2}+2\right) N} \\
& =C_{1} C_{0}^{2} t R^{2} 2^{\left(-\frac{n}{2}+4\right) N}
\end{aligned}
$$

Then, let $k \geq 2$ and assume that

$$
\left\|U_{\ell}\left[\phi_{N, R}\right]\right\|_{\left(M_{2,1}\right)_{N}} \leq C_{0}^{\ell} C_{1}^{\ell-1} t^{\ell-1} R^{\ell} 2^{\left(2 \ell-\frac{n}{2}\right) N},
$$

for all $\ell=1,2, \cdots, k-1$. It is possible to show (5.6) for the case $l=k$ by the assumption (5.6) in a similar way to the proof of Proposition 3.1 .

For the initial data $\phi_{N}$ defined by

$$
\phi_{N}:=R \psi_{N}
$$

we consider the solution

$$
u_{N}(t)=\sum_{k=1}^{\infty} U_{k}\left[\phi_{N}\right](t)
$$

with $u_{N}(0)=\phi_{N}$. Then, we have

$$
\left\|u_{N}(t)\right\|_{H^{s_{*}}} \geq\left\|U_{2}\left[\phi_{N}\right]\right\|_{H^{s_{*}}}-\left\|U_{1}\left[\phi_{N}\right]\right\|_{H^{s_{*}}}-\sum_{k \geq 3}\left\|U_{k}\left[\phi_{N}\right]\right\|_{H^{s_{*}}} .
$$

Since

$$
\operatorname{supp} \widehat{U_{k}\left[\phi_{N}\right]} \subset\left\{\xi \in \mathbb{R}^{n}|| \xi \mid \leq k 2^{N+1}\right\}
$$

and

$$
\widehat{U_{k}\left[\phi_{N}\right]}(t)=\sum_{k_{1}+k_{2}=k} \int_{0}^{t} e^{-i(t-\tau)|\xi|^{2}} \widehat{\left.U_{k_{1}\left[\phi_{N}\right.}\right]}(\tau) * \widehat{U_{k_{2}}\left[\phi_{N}\right]}(\tau) d \tau
$$

we have on the estimate of $U_{k}\left[\phi_{N}\right]$ for $k \geq 3$ from Hölder's inequality and HausdorffYoung's inequality that

$$
\begin{aligned}
\left\|U_{k}\left[\phi_{N}\right]\right\|_{H^{s_{*}}} & \left.\leq\left\|\langle\cdot\rangle^{s_{*}}\right\|_{L^{2}\left(|\xi| \leq k 2^{N+1}\right)} \sup _{|\xi| \leq k 2^{N+1}} \mid \widehat{U_{k}\left[\phi_{N}\right.}\right](\xi) \mid \\
& \left.\leq C(N+\log k)^{\frac{1}{2}} \sum_{k_{1}+k_{2}=k} \int_{0}^{t} \| \widehat{U_{k_{1}}\left[\phi_{N}\right]}\right]\left\|_{L^{2}}\right\| \widehat{U_{k_{2}}\left[\phi_{N}\right]} \|_{L^{2}} d \tau
\end{aligned}
$$


Noticing $\left(M_{2,1}\right)_{N} \subset L^{2}$ and (5.5) , we have

$$
\begin{aligned}
&\left\|U_{k}\left[\phi_{N}\right]\right\|_{H^{s_{*}}} \leq C_{M}(N+\log k)^{\frac{1}{2}} \int_{0}^{t} \sum_{k_{1}+k_{2}=k}\left\|U_{k_{1}}\left[\phi_{N}\right]\right\|_{\left(M_{2,1}\right)_{N}}\left\|U_{k_{2}}\left[\phi_{N}\right]\right\|_{\left(M_{2,1}\right) N} d \tau \\
& \leq C_{M}(N+\log k)^{\frac{1}{2}} \sum_{k_{1}+k_{2}=k} \int_{0}^{t} C^{k_{1}} C_{M}^{k_{1}-1} R^{k_{1}} \tau^{k_{1}-1} 2^{\left(2 k_{1}-\frac{n}{2}\right) N} \\
& \times C^{k_{2}} C_{M}^{k_{2}-1} R^{k_{2}} \tau^{k_{2}-1} 2^{\left(2 k_{2}-\frac{n}{2}\right) N} d \tau \\
&=C^{k} C_{M}^{k-1}(N+\log k)^{\frac{1}{2}} R^{k} t^{k-1} 2^{2\left(k-\frac{n}{2}\right) N} .
\end{aligned}
$$

Then, we take $t$ as $t=2^{-2 N-10}$ and we have the following absolute convergence of the sum for sufficiently large $N$ :

$$
\begin{aligned}
\sum_{k \geq 3}\left\|U_{k}\left[\phi_{N}\right]\right\|_{H^{s_{*}}} & \leq C^{3} C_{M}^{2} 2^{2\left(3-\frac{n}{2}\right) N} R^{3} t^{2} \sum_{k \geq 3}(N+\log k)^{\frac{1}{2}}\left(C C_{M} 2^{2 N} R t\right)^{k-3} \\
& \leq C^{3} C_{M}^{2} R\left(2^{2 N} R t\right)^{2} \sum_{k \geq 3}(N+\log k)^{\frac{1}{2}}\left(C C_{M} 2^{2 N} R t\right)^{k-3}
\end{aligned}
$$

We then let $T_{N} \leq\left(C C_{M} 2^{2 N}\right)^{-1}$ with $R=N^{-1 / 4} \log N$ to obtain that

$$
\sum_{k \geq 3}\left\|U_{k}\left[\phi_{N}\right]\right\|_{H^{s_{*}}} \leq C \frac{(\log N)^{3}}{N^{3 / 4}} \sum_{k \geq 3}(N+\log k)^{\frac{1}{2}}\left(\frac{\log N}{N^{1 / 4}}\right)^{k-3} \rightarrow 0 \quad \text { as } N \rightarrow \infty .
$$

Then, we have from (5.7) with (5.3), (5.4) and (5.10) that

$$
\begin{aligned}
& \left\|u_{N}\left(T_{N}\right)\right\|_{H^{s_{*}}} \geq c T_{N} 2^{2 N} R^{2} N^{\frac{1}{2}}-C R-C \frac{(\log N)^{3}}{N^{3 / 4}} \sum_{k \geq 3}(N+\log k)^{\frac{1}{2}}\left(\frac{\log N}{N^{1 / 4}}\right)^{k-3} \\
& \quad \geq c \log N-C N^{-1 / 4} \log N-C \frac{(\log N)^{3}}{N^{1 / 4}} \sum_{k \geq 3}\left(1+N^{-1} \log k\right)^{\frac{1}{2}}\left(\frac{\log N}{N^{1 / 4}}\right)^{k-3} \\
& \rightarrow \infty \text { as } N \rightarrow \infty
\end{aligned}
$$

On the other hand, we have

$$
\left\|\phi_{N}\right\|_{H^{s_{*}}} \leq C \frac{\log N}{N^{\frac{1}{4}}} \rightarrow 0 \quad \text { as } N \rightarrow \infty
$$

This completes the proof of the ill-posedness in $H^{-1}\left(\mathbb{R}^{2}\right)$.

Remark. If we use the nonscaled modulation space $M_{2,1}=\left.\left(M_{2,1}\right)_{N}\right|_{N=1}$, then there appears some regularity loss, and the estimate in (5.8) loses the gain of the growth order. This prevents us from showing the ill-posedness for the critical case. 


\section{ACKNOWLEDGMENT}

The work of the second author was partially supported by JSPS Grant-in-Aid, Scientific Research A \#20244009 and Scientific Research S \#25220702.

\section{REFERENCES}

[1] Ioan Bejenaru and Daniela De Silva, Low regularity solutions for a 2D quadratic nonlinear Schrödinger equation, Trans. Amer. Math. Soc. 360 (2008), no. 11, 5805-5830, DOI 10.1090/S0002-9947-08-04415-2. MR2425692 (2009k:35289)

[2] Ioan Bejenaru and Terence Tao, Sharp well-posedness and ill-posedness results for a quadratic non-linear Schrödinger equation, J. Funct. Anal. 233 (2006), no. 1, 228-259, DOI 10.1016/j.jfa.2005.08.004. MR2204680(2007i:35216)

[3] Daniella Bekiranov, The initial-value problem for the generalized Burgers' equation, Differential Integral Equations 9 (1996), no. 6, 1253-1265. MR1409926 (97k:35219)

[4] Thierry Cazenave and Fred B. Weissler, The Cauchy problem for the critical nonlinear Schrödinger equation in $H^{s}$, Nonlinear Anal. 14 (1990), no. 10, 807-836, DOI 10.1016/0362546X(90)90023-A. MR1055532(91j:35252)

[5] J. E. Colliander, J.-M. Delort, C. E. Kenig, and G. Staffilani, Bilinear estimates and applications to $2 D$ NLS, Trans. Amer. Math. Soc. 353 (2001), no. 8, 3307-3325 (electronic), DOI 10.1090/S0002-9947-01-02760-X. MR.1828607 (2002d:35186)

[6] Daniel B. Dix, Nonuniqueness and uniqueness in the initial-value problem for Burgers' equation, SIAM J. Math. Anal. 27 (1996), no. 3, 708-724, DOI 10.1137/0527038. MR1382829 (97c:35174)

[7] H. G. Feichtinger, Modulation spaces on locally compact Abelian groups, Technical Report, University of Vienna, 1983, in: "Proc. Internat. Conf. on Wavelets and Applications" (Radha, R.; Krishna, M.; Yhangavelu, S., eds.), New Delhi Allied Publishers, 2003, 1-56.

[8] J. Ginibre and G. Velo, On a class of nonlinear Schrödinger equations. II. Scattering theory, general case, J. Funct. Anal. 32 (1979), no. 1, 33-71, DOI 10.1016/0022-1236(79)90077-6. MR.533219 (82c:35058)

[9] Tosio Kato, On nonlinear Schrödinger equations (English, with French summary), Ann. Inst. H. Poincaré Phys. Théor. 46 (1987), no. 1, 113-129. MR877998 (88f:35133)

[10] Carlos E. Kenig, Gustavo Ponce, and Luis Vega, Quadratic forms for the 1-D semilinear Schrödinger equation, Trans. Amer. Math. Soc. 348 (1996), no. 8, 3323-3353, DOI 10.1090/S0002-9947-96-01645-5. MR1357398 (96j:35233)

[11] Carlos E. Kenig, Gustavo Ponce, and Luis Vega, On the ill-posedness of some canonical dispersive equations, Duke Math. J. 106 (2001), no. 3, 617-633, DOI 10.1215/S0012-709401-10638-8. MR.1813239 (2002c:35265)

[12] Nobu Kishimoto, Local well-posedness for the Cauchy problem of the quadratic Schrödinger equation with nonlinearity $\bar{u}^{2}$, Commun. Pure Appl. Anal. 7 (2008), no. 5, 1123-1143, DOI 10.3934/cpaa.2008.7.1123. MR2410871 (2009c:35442)

[13] Nobu Kishimoto, Low-regularity bilinear estimates for a quadratic nonlinear Schrödinger equation, J. Differential Equations 247 (2009), no. 5, 1397-1439, DOI 10.1016/j.jde.2009.06.009. MR2541415 (2011b:35488)

[14] Luc Molinet, Sharp ill-posedness results for the KdV and mKdV equations on the torus, Adv. Math. 230 (2012), no. 4-6, 1895-1930, DOI 10.1016/j.aim.2012.03.026. MR2927357

[15] Luc Molinet and Stéphane Vento, Sharp ill-posedness and well-posedness results for the KdVBurgers equation: the real line case, Ann. Sc. Norm. Super. Pisa Cl. Sci. (5) 10 (2011), no. 3, 531-560. MR2905378 (2012k:35484)

[16] Seungly Oh and Atanas Stefanov, On quadratic Schrödinger equations in $\mathbf{R}^{1+1}$ : a normal form approach, J. Lond. Math. Soc. (2) 86 (2012), no. 2, 499-519, DOI 10.1112/jlms/jds016. MR2980922

[17] Gustavo Ponce and Thomas C. Sideris, Local regularity of nonlinear wave equations in three space dimensions, Comm. Partial Differential Equations 18 (1993), no. 1-2, 169-177, DOI 10.1080/03605309308820925. MR.1211729 (95a:35092)

[18] Joachim Toft, Continuity properties for modulation spaces, with applications to pseudo-differential calculus. I, J. Funct. Anal. 207 (2004), no. 2, 399-429, DOI 10.1016/j.jfa.2003.10.003. MR2032995 (2004j:35312) 
[19] Yoshio Tsutsumi, $L^{2}$-solutions for nonlinear Schrödinger equations and nonlinear groups, Funkcial. Ekvac. 30 (1987), no. 1, 115-125. MR.915266 (89c:35143)

[20] Wang Baoxiang, Zhao Lifeng, and Guo Boling, Isometric decomposition operators, function spaces $E_{p, q}^{\lambda}$ and applications to nonlinear evolution equations, J. Funct. Anal. 233 (2006), no. 1, 1-39, DOI 10.1016/j.jfa.2005.06.018. MR2204673(2007b:35187)

Department of Mathematics, Chuo University, Kasuga, Bunkyoku, Tokyo, 112-8551 JAPAN

Mathematical Institute, Tohoku University, Sendai 980-8578, Japan 